\title{
Génétique inverse et mucoviscidose
}

La mucoviscidose est la plus fréquente des maladies héréditaires. L'approche de la "génétique inverse», allant du gène vers ce pour quoi il code et non l'inverse, a mis sur la piste du gène qui est modifié chez les malades. Le diagnostic prénatal de l'affection est d'ores et déjà possible et la prochaine identification de la protéine anormale en cas de mucoviscidose permettra probablement d'en comprendre le mécanisme et peut-être de proposer des traitements nouveaux.

\section{Alain Kitzis \\ Chef de travaux au CHU Cochin Paule Warren \\ Licencièe ès sciences, université Laval, Québec \\ Jean-Claude Kaplan Professeur au CHU Cochin}

\section{ADRESSE}

A. Kitzis, P. Warren, J.-C. Kaplan : institut de pathologie moléculaire, CHU Cochin, 24, rue du Faubourg-Saint-Jacques, 75014 Paris, France.

\section{TRÉS A PART}

A. Kitzis : institut de pathologie moléculaire, CHU Cochin, 24, rue du Faubourg-SaintJacques, 75014 Paris, France.

$\mathrm{m} / \mathrm{s} n^{\circ} 3$ vol. 4, mars 88 a mucoviscidose ou fibrose kystique du pancréas (CF pour cystic fibrosis des anglosaxons) est la maladie héréditaire la plus fréquente dans les populations d'origine européenne. Un enfant sur 1600 à 2000 naît atteint de cette maladie. L'affection est transmise selon un mode autosomique récessif, et environ une personne sur 20 est porteuse du gène morbide ${ }^{*}$, soit près de trois millions de personnes en France.

Il s'agit d'une exocrinopathie touchant essentiellement les voies respiratoires et digestives: un épaississement des sécrétions entraîne des phénomènes d'obstruction touchant les canaux excréteurs.

La lésion moléculaire de la mucoviscidose demeure totalement inconnue et, aucune thérapeutique efficace n'étant disponible, seuls sont mis en ouvre des traitements symptomatiques à visée palliative: kinésithérapie, antibiothérapie ou encore enzymes pancréatiques de substitution (lipase et amylase).

Le diagnostic de l'affection repose sur un tableau clinique fait de manifestations pulmonaires et digestives, ces dernières pouvant être très précoces au point de revêtir l'aspect d'un iléus méconial ( 10 à $15 \%$ des nouveaunés mucoviscidosiques). Il est étayé par des tests biologiques: dosage de la trypsinémie, et surtout test à la sueur dont la fiabilité est quasi absolue. En revanche les héterrozygotes sont cliniquement et biologiquement normaux et, à l'heure actuelle, il n'existe aucun moyen de les dépister s'ils n'ont déjà eu un enfant atteint de mucoviscidose. En l'absence de traitement efficace, plus de la moitié des enfants mucoviscidosiques meurent avant l'âge de 15-20 ans. A défaut de moyens de détection des hétérozygotes, la seule attitude en vigueur était, jusqu'en 1983, le conseil génétique classique, consistant en une explication du risque a priori dans les familles qui ont déjà eu un enfant atteint, c'est-à-dire une chance sur quatre à chaque naissance.

* La mucoviscidose touche un nouveau-né sur 1 600. En appliquant la loi de distribution binomiale d'Hardy-Weinberg $\left(p^{2}+2 p q+q^{2}\right.$ $=1$, où $p$ et $q$ représentent la fréquence respective de deux allèles exclusifs, $p^{2}$ et $q^{2}$ expriment la fréquence des homozygotes et $2 p q$ celle des hétérozygotes), on calcule la fréquence du gène $\mathrm{CF}=1 / 40$, et celle des individus porteurs $=1120$. 


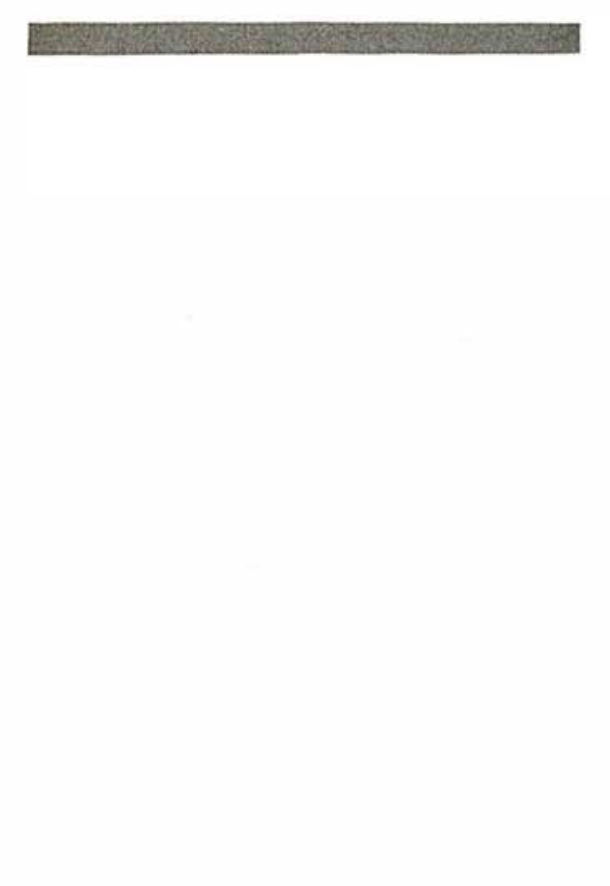

RÉFÉRENCES

1. Brock DJH. Amniotic fluid alkaline phosphatase isoenzyme in early prenatal diagnosis in cystic fibrosis. Lancet 1983 ; ii : 941-3.

2. Muller F, Berg S, Frot JC, Boue J, Boue A. Prenatal diagnosis of cystic fibrosis. I. Prospective of 51 pregnencies. Prenat Diagn $1985 ; 5: 97-108$.

3. Botstein D, White RL, Skolnick M, Davis RW. Construction of a genetic linkage map in man using restriction fragment length polymorphisms. Am J Human Genet 1980 ; 32 : 314-31.

4. Eiberg H, Mohr J, Schmiegelow K, Nielsen LS, Williamson $R$. Linkage relationships of paraoxonase (PON) with other markers: indication of PON-cystic fibrosis synteny. Clin Genet 1985 ; 28 : 265-71.

5. Tsui LC, Buchwald M, Barker D, et al. Cystic fibrosis locus defined by a genetically linked polymorphic DNA marker. Science $1985 ; 230$ : 1054-7.

6. Knowlton RG, Cohen-Haguenauer $\mathrm{O}$, Cong NV, et al. A polymorphic DNA marker linked to cystic fibrosis is located on chromosome 7 . Nature $1985 ; 318: 380-2$.

7. Wainwright BJ, Scambler PJ, Schmidtke $\mathrm{J}$, et al. Localization of cystic fibrosis locus to human chromosome 7cenq22. Nature $1985 ; 318: 384-5$.

8. White R, Woodward S, Leppert M, et al. A closely linked genetic marker for cystic

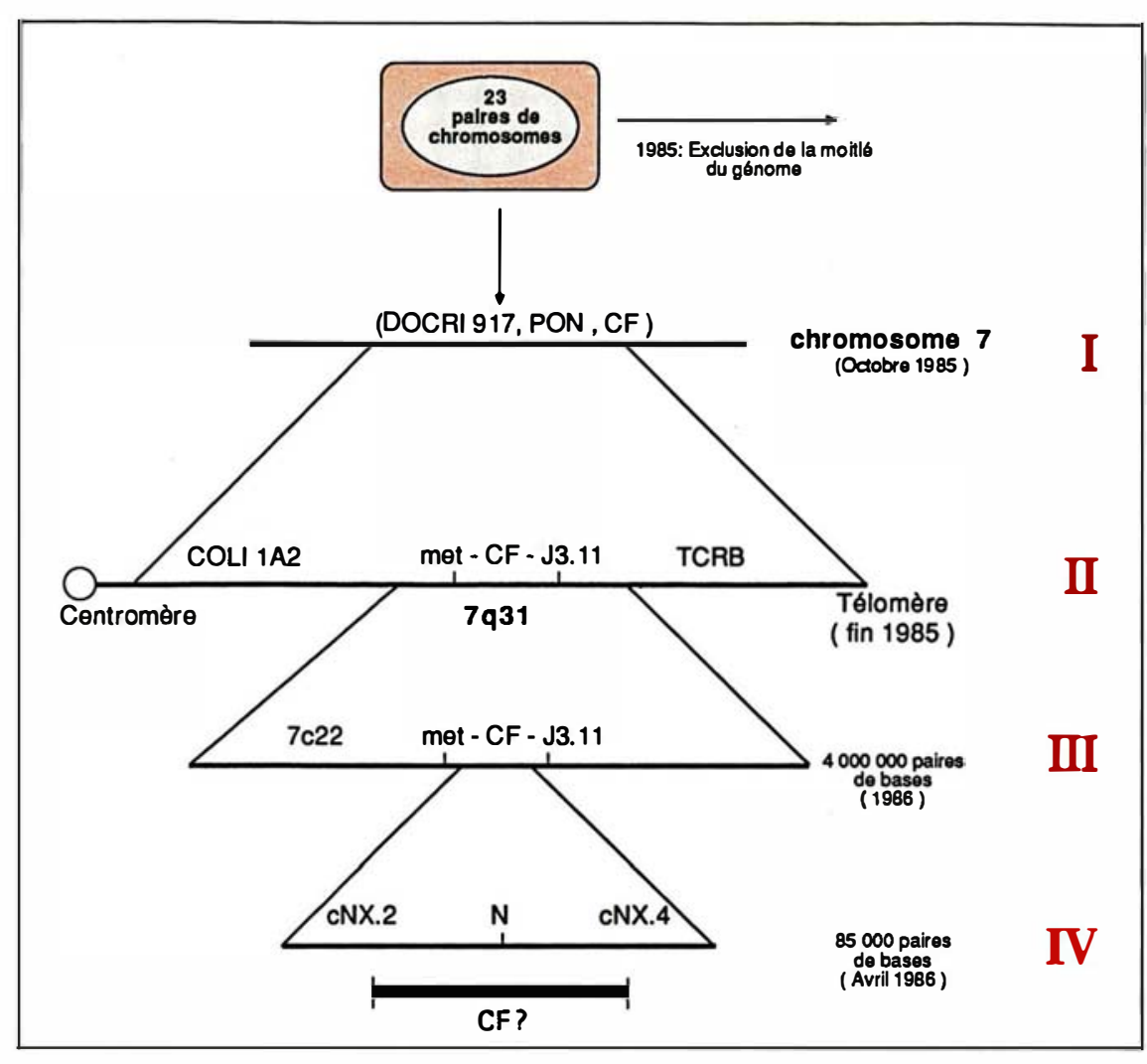

Figure 1. Les étapes de la recherche du gène de la mucoviscidose. Les positions des différents loci définis par les sondes sont indiquées au-dessus des fragments d'ADN. N: site de restriction Not 1.

Depuis 1983 un diagnostic prénatal par études enzymologiques du liquide amniotique est possible [1, 2], fondé sur le dosage des phosphatases alcalines d'origine intestinale, fortement diminuées dans le liquide amniotique des foetus atteints de mucoviscidose. Ce diagnostic a cependant l'inconvénient de ne pouvoir être pratiqué que pendant une fenêtre étroite : la $17^{\mathrm{e}}$ et la $18^{\mathrm{e}}$ semaine d'aménorrhée. Des erreurs portant sur l'estimation de l'âge de la grossesse pourraient expliquer le taux élevé de faux négatifs, compris entre 1 et $10 \%$ selon les laboratoires (A. Beaudet, communication à la conférence nordaméricaine sur la mucoviscidose, Toronto, octobre 1987).

En fait, la génétique moléculaire offre désormais la possibilité de rechercher directement sur le génome les gènes responsables de maladies monofactorielles dues à une lésion d'un gène non encore identifié. Cette démarche a bien entendu été appliquée à la mucoviscidose. Elle recueille ses premiers succès en ce qui concerne le diagnostic prénatal à l'aide de marqueurs génotypiques, et l'arrivée au gène lui-même est en vue.

\section{Localisation}

chromosomique du locus CF

La mucoviscidose fait partie des maladies monofactorielles à gène inconnu où toutes les recherches antérieures visant à identifier une protéine responsable étaient demeurées vaines. En 1980, il est apparu que les innombrables marqueurs génotypiques que sont les polymorphismes de restriction, ou RFLP (restriction fragment length polymorphism) ( $m / s$ suppl. au $n^{\circ} 7$, vol. 3, p. 4) offraient la possibilité de sortir de l'impasse [3]. La stratégie de la génetique inverse consiste: (a) à utiliser ces marqueurs pour des 
études de linkage permettant dans un premier temps de localiser le locus morbide sur un chromosome, puis sur une région chromosomique; (b) à le cerner ; (c) à l'isoler à partir d'un ADN normal; (d) à le cloner et le séquencer ; (e) en fin de compte à déchiffrer la protéine correspondant au message génétique. Cette démarche nouvelle est appelée «génétique inverse » car elle procède d'une stratégie qui est l'inverse de celle de la génétique classique : alors que celle-ci part de la protéine pour arriver au gène (exemples: les hémoglobinopathies, les hémophilies), la génétique inverse part du gène pour descendre vers la protéine.

La première étape a été particulièrement longue à franchir. En effet, en l'absence de tout indice

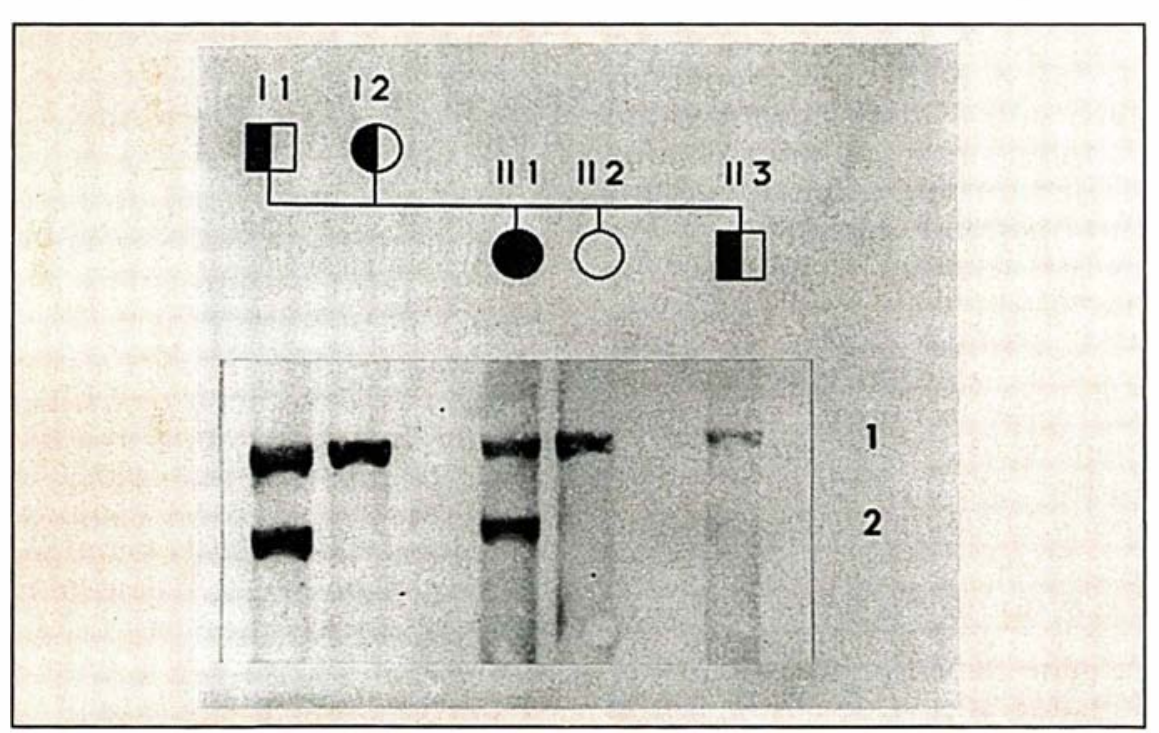

Figure 2. Étude familiale par la sonde met $D$ on vue d'un diagnostic prénatal de mucoviscidose. L'ADN préparé à partir des globules blancs ou des villosités choriales prélevées à la dixième semaine de grossesse a été digéré par l'enzyme Taql, soumis à une électrophorèse en gel d'agarose à $0,8 \%$ et transféré sur un filtre de nylon selon la technique de Southern (I1, père ; 12, mère; II1, enfant mucoviscidosique; 112, enfant normal; II3, prélèvement de trophoblaste). L'enfant mucoviscidosique est hétérozygote pour les allèles 1 et 2. Le père est également hétérozygote pour ces allèles. La mère est homozygote pour l'allèle 1. L'analyse du trophoblaste montre une bande au niveau de l'allèle 1 et pas de bande au niveau de l'allèle 2. Cet allèle, que possède l'enfant mucoviscidosique et qui vient du père, signe le chromosome atteint: le fœtus ne le possède pas et il sera soit homozygote normal, s'il a reçu le chromosome normal de la mère, soit hétérozygote sain, s'il a reçu le chromosome atteint de la mère, ce que cette seule étude ne peut dire. L'utilisation de la sonde $\mathbf{p} \mathbf{3 . 1 1}$ (non représentée ici) a confirmé que le fotus a bien reçu le gène normal du père, ce qui permet d'éliminer le risque de simple recombinaison et a montré qu'il a reçu l'allèle atteint de la mère, ce qui indique qu'il sera donc hétérozygote sain. Ce diagnostic a été confirmé par la naissance d'un enfant sain (trypsinémie normale).

$\mathrm{m} / \mathrm{s} n^{\circ} 3$ vol. 4 , mars 88 au moins deux enfants mucoviscidosiques, et effectuer un calcul statistique de la probabilité de linkage. Lorsqu'un résultat négatif (non liaison) est obtenu pour une sonde donnée, la localisation proche du locus morbide est exclue. La procédure est lourde et, même si le succès est en principe garanti, il est soumis aux lois du hasard. C'est ainsi qu'il a fallu 4 ans pour exclure environ $50 \%$ du genome (R. Williamson, St Mary's Hospital, Londres). Paradoxalement, le premier linkage significatif a été obtenu par une équipe danoise à l'aide d'un marqueur protéique polymorphe : la paraxonase plasmatique* ${ }^{*}$ [4]. Cependant la localisation chromosomique du locus correspondant (locus PON) demeurait inconnue, ce qui réduisait la portée de cette découverte. L'intérêt de ce linkage a été cependant de montrer que dans les 68 familles de mucoviscidosiques étudiées le locus CF paraissait unique.

En cette même année, à l'automne 1985, une étude américano-canadienne montrait enfin une liaison génétique entre une sonde anonyme, Docri 917 et le gène $\mathrm{CF}$ [5] (figure 1). Cette sonde, qui était aussi liée au locus PON, fut ensuite localisée par l'équipe de J. Frézal sur le bras long du chromosome 7 [6]. Simultanément, l'équipe de $\mathrm{R}$. Williamson à Londres et celles de $\mathrm{R}$. White et de G. Vande Woude aux ÉtatsUnis décrivaient deux sondes liées au gène $C F: p J 3.11$ d'une part et le proto-oncogène met d'autre part [7, 8]. L'évaluation des distances génétiques devait ensuite montrer que la sonde Docri 917 était située relativement loin du gène $\mathrm{CF}$ (environ 15 centimorgans), tandis que $\mathrm{pJ} 3.11$ et $m e t$ étaient situées beaucoup plus près (respectivement à 0,3 et 0,4 centimorgan du locus CF). De plus ces sondes étaient situées de part et d'autre de ce locus [9, 10]. On pou-

\footnotetext{
* Enzyme clivant le paroxon, substance anticholinestérasique utilisée dans le traitement du glaucome et dont il existe un polymorphisme phénotypique.
} 
. Park M, Blair DG, et al. gene from a chemically transformed human cell line. Nature $1984 ; 311: 29-33$

13. Cooper CS, Blair DG, Oskarsson MK, Tainsky MA, Eader LA, Vande Woude G. Characterization of human transforming genes from chemically transformed teratocarcinoma and pancreatic carcinoma cell lines. Cancer Res 1984 ; 44 : 1-10.

14. Scambler PJ, Law HY, Williamson $R$, Cooper CS. Chromosone mediated gene transfer of six DNA markers linked to the cystic fibrosis locus on human chromosome seven. Nucleic Acids Res 1986 ; 14 : 7159-74.

15. Estivill X, Farrall M, Scambler PJ, et al. A candidate for the cystic fibrosis locus isolated by selection for methylation-free islands. Nature 1987 ; 326 : 840-5.

16. Bird AP. CpG-rich islands and the function of DNA methylation. Nature 1986 ; 321 : vait dès lors considérer que ce dernier était cerné dans un domaine d'ADN d'environ $1 \mathrm{Mb}$. Dès lors les recherches se sont orientées simultanément dans deux directions: d'une part, la mise en ouvre immédiate d'un diagnostic prénatal génotypique, d'autre part la quête du gène $\mathrm{CF}$ lui-même.

\section{Diagnostic prénatal et sondes indirectes}

Les sondes $\mathrm{pJ} 3.11$, metD ou $m e t H$ déf in issent $5 \mathrm{R} F \mathrm{LP}$ (figure 2, page 153). Leur application au diagnostic prénatal était possible en raison: (a) de leur proximité, ce qui minimise les risques de recombinaison; (b) de leur situation de part et d'autre du locus $C F$, ce qui augmente considérablement la fiabilité de la méthode, en permettant de visualiser à coup sûr les recombinaisons simples, dont la probabilité est inférieure à $1 \%$ pour chaque RFLP ; (c) de l'informativité qui est importante, puisque $77 \%$ des couples ayant un enfant mucoviscidosique vivant sont informatifs ${ }^{* *}$, parmi lesquels $55 \%$ le sont des deux côtés du gène $\mathrm{CF}$. C'est par cette technique que nous avons réalisé notre premier diagnostic prénatal de mucoviscidose en 1986 (figure 2).

\section{La quête du gène}

par d'autres moyens. Ceux-ci ont été fournis par le procédé dit de chromosome mediated gene transfer (CMGT) (voir nouvelle $\mathrm{m} / \mathrm{s}$ $n^{\circ} 1$, vol. 3, p. 47) [11]. Il consiste à transfecter in vitro des chromosomes métaphasiques dans une cellule hôte appartenant à une espèce différente. En fait les chromosomes ne demeurent pas entiers lors de la manipulation et ce sont des fragments qui pénètrent dans la cellule réceptrice. Ce système réclame la présence d'un marqueur qui permet la sélection positive de la cellule ayant incorporé le fragment recherché. En ce qui concerne le gène $C F$, Williamson a eu l'idée que l'oncogène met pourrait justement constituer un tel marqueur, en raison de sa localisation providentiellement proche. Il a donc utilisé comme source de chromosome humain une lignée d'ostéosarcome portant une translocation chromosomique $t(1 ; 7)$ dans laquelle on savait que met était activé, donc capable de transformer in vitro les cellules de la lignée de souris NIH 3T3 [12, 13]. C'est ainsi qu'a été obtenue la lignée CII, où les cellules transformées par met activé se sont avérées contenir de l'ADN humain hybridant aussi avec la sonde $\mathrm{pJ} 3.11$ [14]. Le gène $C F$ étant situé entre ces deux séquences (figure 1), l'opération de transfert du gène $C F$ dans une cellule de souris était réussie. Ceci équivaut à un clonage moléculaire, avec une nuance importante: le fragment d'ADN humain cloné ne peut être séparé en bloc de l'ADN de souris, à la différence de ce qui se passe dans un clonage classique. Or le fragment intéressant représente environ $4 \mathrm{Mb}$ (figure 1, étape III), ultérieurement réduits à $\mathrm{l} \mathrm{Mb}$ dans le sous-clone cellulaire CIId renfermant toujours met. C'est dans ce dernier que Williamson a fait le pari que se trouvait le gène $C F$.

\section{Le saut vers le gène CF}

* L'informativité résulte de l'hétérozygotie d'un RFLP permettant de distinguer les deux chromosomes d'une même paire et d'attribuer un locus morbide à l'un des deux.
L'ADN de la lignée CIId a été cloné dans une banque de cosmides où les rares clones d'ADN 


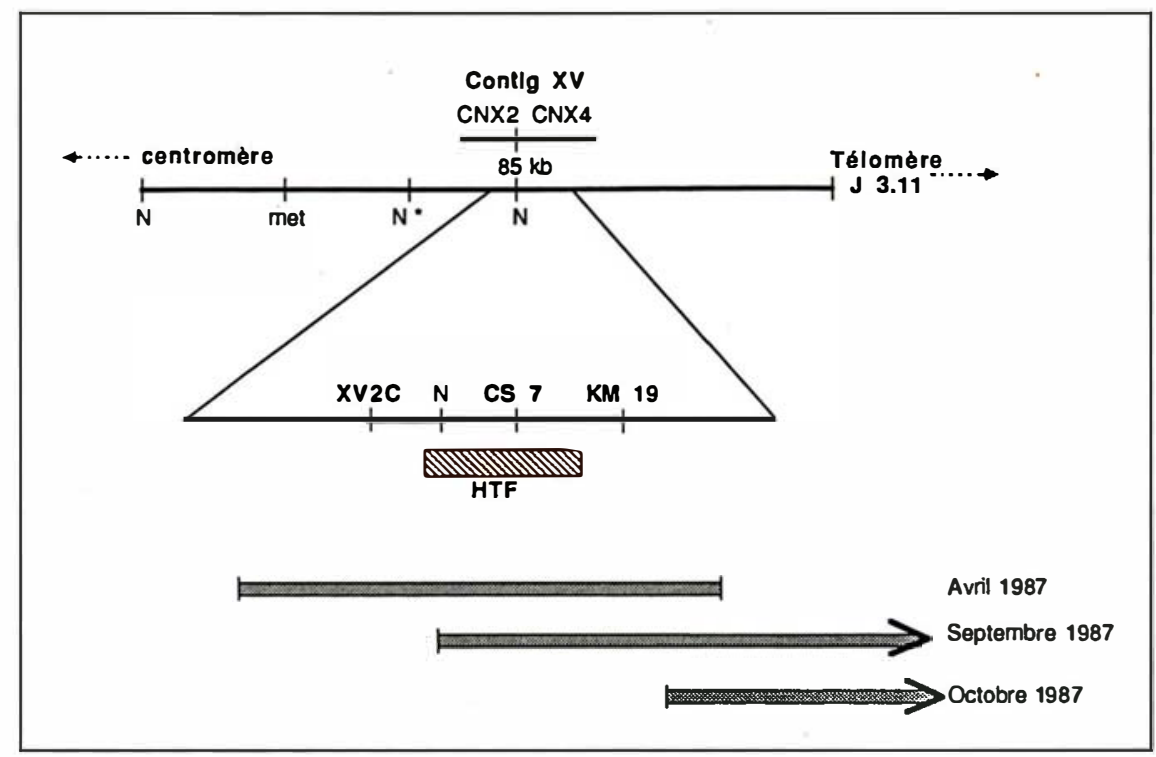

Figure 3. Caractérisation du segment d'ADN susceptible de contenir le gène CF. Représentation du fragment d'ADN de 85 kilobases (kb) (contig), centré sur le site Not I $(N)$, et situé à environ $650 \mathrm{~kb}$ du locus met. Le site Not I facultatif, situé entre met et XV 2c, est représenté par $N^{*}$. La localisation proposée du locus CF à différentes étapes de sa recherche est représentée par les rectangles gris.

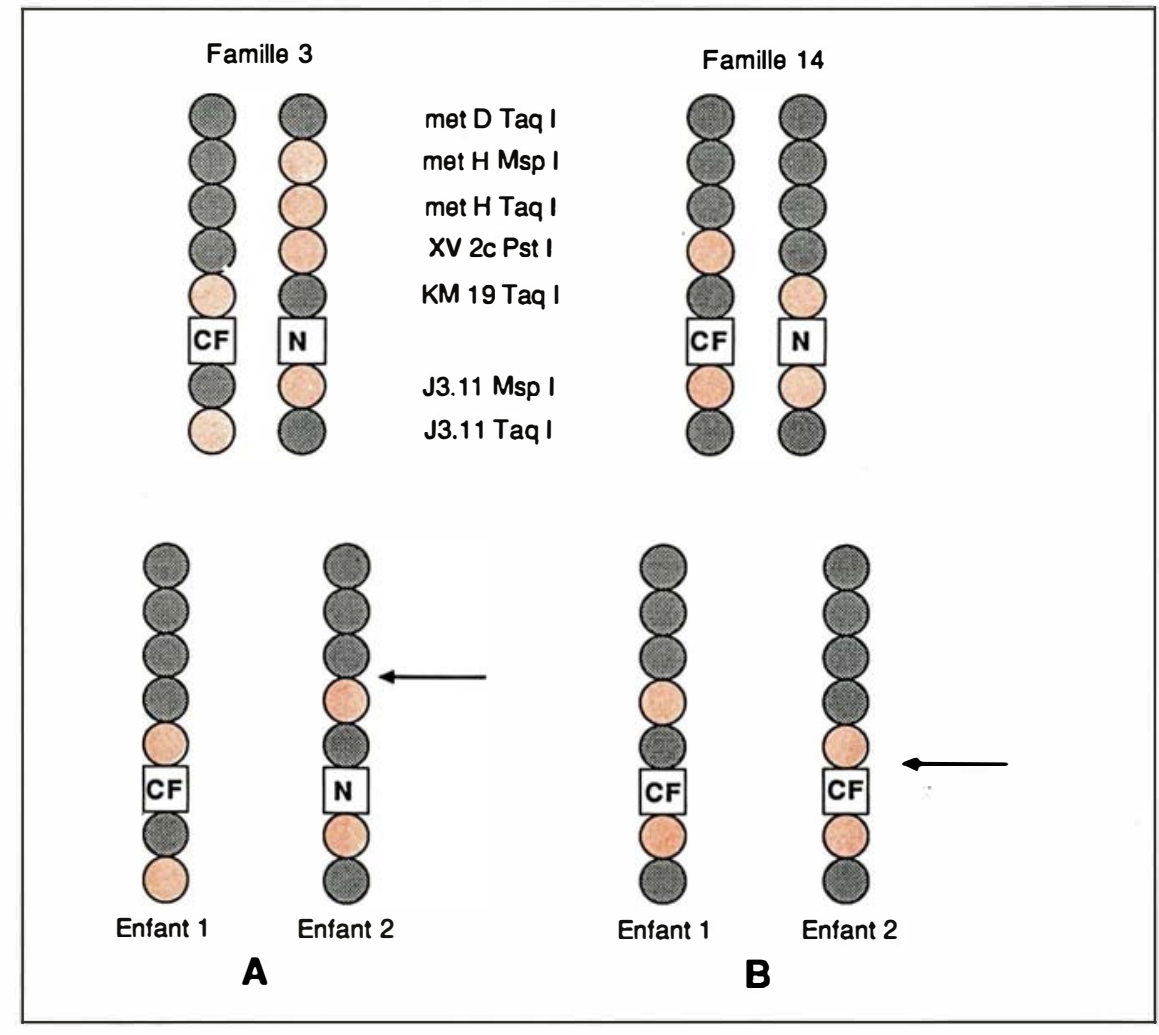

Figure 4. Représentation schématique des crossing-over proches du locus CF. (A), entre les sondes met et le locus CF; (B), entre les sondes XV2CKM19 et le locus. La flèche indique le lieu du crossing-over chez le deuxième enfant des familles 3 et 14 (résultats personnels non publiés). Grands allèles = cercles gris ; petits allèles $=$ cercles rouges.

$m / s n^{\circ} 3$ vol. 4 , mars 88 humain ont été repérés, puis utilisés pour cribler une banque spécifique du chromosome 7 humain. Ceci a permis de repérer deux cosmides contigus, car hybridant avec une même sonde. Cette stratégie dite des contigs consiste à reconstituer une longue séquence d'ADN génomique à partir de clones chevauchants et ordonnés (figure 1, étape IV et figure 3). Grâce à la méthode d'électrophorèse en champ pulsé, le contig XV, constitué par les deux cosmides CNX2 et CNX4 partageant le même site Notl (figure 3), a été localisé à environ 650-700 kilobases $(\mathrm{kb})$ du gène met [15]. L'étape suivante a consisté à rechercher systématiquement les régions riches en doublet $\mathrm{CpG}$ dont la cytosine n'est pas méthylée, ce qui définit des ilôts dits HTF (HpaII tiny) fragments) qui caractérisent les régions de l'ADN renfermant des gènes activement transcrits dans tous les types cellulaires [16]. Le clone génomique CS7 répondait à ces critères (figure 3 ).

\section{Le gène de la mucoviscidose?}

Comment démontrer que la région CS7 est le bon candidat ? Les critères suivants ont été rassemblés [15] : (a) séquence conservée dans le règne animal (technique dite du zoo-blotting); (b) séquence exprimée dans un grand nombre de tissus dont le poumon; (c)présence d'un RFLP ne recombinant plus avec le locus CF dans les familles où une recombinaison avait été observée avec les sondes met et pJ 3.11 (figure 4). Deux nouveaux RFLP du contig XV, définis par les sondes XV2c et KMI9 situées de part et d'autre de CS7 (figure 3), ont également montré leur plus grande proximité génétique du locus CF, notamment dans une famille que nous avons étudiée (figure 4A).

Ces critères, tous indirects, ne sont cependant pas suffisants pour affirmer que le gène est atteint. La preuve définitive, et non encore apportée, serait de démontrer que 


\begin{tabular}{|c|c|c|c|c|c|c|}
\hline \multicolumn{7}{|c|}{$\begin{array}{l}\text { Tableau I } \\
\text { TION DES HAPLOTYPES CONSTITUÉS } \\
\text { R DES SONDES pXV2c et pCS7 [15] }\end{array}$} \\
\hline \multicolumn{7}{|c|}{$\begin{array}{l}\text { Les allèles } 1 \text { et } 2 \text { codent respectivement pour les grands et les petits fragment } \\
\text { de restriction définis pour chaque couple sonde/enzyme. }\end{array}$} \\
\hline Haplotypes & $\begin{array}{l}\text { pXV-2c } \\
\text { Taq } 1\end{array}$ & $\begin{array}{l}\text { pCS.7 } \\
\text { Hha } 1\end{array}$ & Normaux & $(\%)$ & CF & (\%) \\
\hline $\begin{array}{l}\mathbf{A} \\
\mathbf{B} \\
\mathbf{C} \\
\mathbf{D}\end{array}$ & $\begin{array}{l}1 \\
1 \\
2 \\
2\end{array}$ & $\begin{array}{l}1 \\
2 \\
1 \\
2\end{array}$ & $\begin{array}{c}9 \\
22 \\
28 \\
6\end{array}$ & $\begin{array}{c}14 \\
34 \\
43 \\
9\end{array}$ & $\begin{array}{l}0 \\
66 \\
0 \\
4\end{array}$ & $\begin{array}{l}0 \\
94 \\
0 \\
6\end{array}$ \\
\hline Total & & & 65 & 100 & 70 & 100 \\
\hline
\end{tabular}

le gène candidat est muté chez les mucoviscidosiques. Nos résultats très récents et non encore publiés ont cependant montré l'existence d'une recombinaison entre KM19-XV2c et le locus CF (figure $4 B$ ), ce qui exclut le gène candidat CS7. Ceci illustre bien la difficulté de l'entreprise lorsque l'on ne dispose pas d'un fil conducteur*. Le gène $C F$ ne doit pas être loin de la région étudiée, et même si l'absence d'indice complique singulièrement la tache en rendant nécessaire un fastidieux travail de séquençage dans un génome mucoviscidosique, le succès est garanti à terme.

\section{Un résultat inattendu}

La mutation CF résulterait d'un effet fondateur. Le travail publié par l'équipe de $\mathrm{R}$. Williamson en avril 87 [15] contient en outre des résultats tendant à prouver une origine monocentrique de la mutation CF. En effet, dans la population d'Europe du Nord, il

\footnotetext{
- Rappelons que dans le cas de la myopathie de Duchenne l'existence de délétions a représenté un atout majeur.
}

- Nos résultats préliminaires tendent à montrer que ce déséquilibre de liaison existe aussi existe un déséquilibre de liaison significatif entre un certain haplotype et le gène $C F$ muté (Tableau I): l'haplotype B est retrouvé dans $94 \%$ des chromosomes portant l'anomalie CF et $34 \%$ des chromosomes normaux, alors que l'haplotype C est retrouvé dans $0 \%$ des chromosomes portant CF et $43 \%$ des normaux. Ce très fort déséquilibre de liaison est en faveur d'un événement fondateur récent. Il reste à déterminer si cet événement est unique ou s'il est survenu indépendamment dans des populations différentes ${ }^{* *}$. L'existence d'un même déséquilibre de liaison dans différentes populations d'Europe serait très en faveur d'un fondateur originel unique, et donc d'une mutation univoque. Cette découverte est d'une portée considérable, d'une part parce que l'existence d'un allèle pathologique unique permet d'envisager un diagnostic prénatal génotypique, et à terme phénotypique (lorsque le produit du gène sera identifié) considérablement simplifié, d'autre part parce que, pour la première fois, une détection pourrait être envisagée chez les porteurs sains, sans réclamer le «préavis » d'un cas index. Il reste à expliquer la remarquable dissémination de ce gène pathologique dans la population européenne, laquelle suggère un avantage sélectif des hétérozygotes

\section{Summary}

Cystic fibrosis (CF) is one of the most frequent autosomal recessive disorder in man. One child is affected among 1600 newborns; one individual among $20-25$ is a silent heterozygous carrier. Classical biochemical methods have failed to uncover the abnormal defective gene product, and the responsible gene is not known. Molecular genetics gives us now the possibility to reach this unknown gene, to read its sequence and to deduce the nature and the function of the corresponding protein. The molecular pathology of the abnormal gene will thus be deciphered, and ultimately the malfunction of the affected cells understood. This strategy, going from the disease to the gene and then to its function is called « reverse genetics». We describe the different steps of this search: (1) localization of the morbid locus, called CF, on chromosome 7 ; (2) regional mapping of the $\mathrm{CF}$ locus ; (3) approaching the CF gene itself. The final goal, ie reaching and deciphering the $\mathrm{CF}$ gene, is in view but not reached yet. Nonetheless several useful restriction fragment length polymorphisms (RFLP) closely linked to the CF locus, allow us already to perform a safe prenatal detection of foetuses at risk. A strong linkage disequilibrium between a particular haplotype and the abnormal CF gene was unexpectedly found in Northern - and Western Europe, suggesting that a same abnormal founding allele has widespread in these populations.

\section{Remerciements}

Paule Warren est boursière de l'association française de lutte contre la mucoviscidose. 